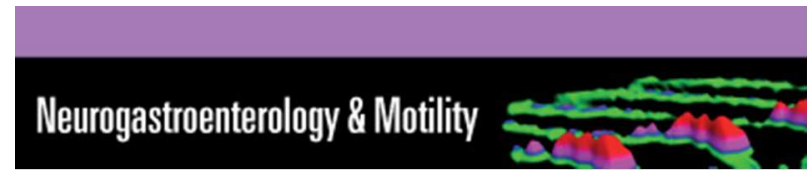

\title{
Absence of intestinal inflammation and postoperative ileus in a mouse model of laparoscopic surgery
}

\begin{tabular}{|c|c|}
\hline Journal: & Neurogastroenterology and Motility \\
\hline Manuscript ID: & NMO-00378-2013 \\
\hline Manuscript Type: & Original Article \\
\hline Date Submitted by the Author: & 17-Dec-2013 \\
\hline Complete List of Authors: & $\begin{array}{l}\text { Gomez-Pinilla, Pedro; KU Leuven, Translational Research in } \\
\text { GastroIntestinal Disorders (TARGID) and Department of Clinical and } \\
\text { Experimental Medicine } \\
\text { Binda, Mercedes; KU Leuven, Laboratory Abdominal Surgical Oncology, } \\
\text { Department of Oncology } \\
\text { Lissens, Ann; KU Leuven, Centre for Surgical Technologies } \\
\text { DiGiovangiulio, Martina; KU Leuven, Translational Research in } \\
\text { GastroIntestinal Disorders (TARGID) and Department of Clinical and } \\
\text { Experimental Medicine } \\
\text { van Bree, Sjoerd H; Academic Medical Center, Tytgat Institute of Liver and } \\
\text { Intestinal Research } \\
\text { Nemethova, Andrea; University of Leuven, Division of Gastroenterology, } \\
\text { TARGID, Translational Research Center for Gastrointestinal Disorders } \\
\text { Stakenborg, Nathalie; KU Leuven, Translational Research in } \\
\text { GastroIntestinal Disorders (TARGID) and Department of Clinical and } \\
\text { Experimental Medicine } \\
\text { Farro, Giovanna; KU Leuven, Translational Research in GastroIntestinal } \\
\text { Disorders (TARGID) and Department of Clinical and Experimental Medicine } \\
\text { Bosmans, Goele; KU Leuven, Translational Research in GastroIntestinal } \\
\text { Disorders (TARGID) and Department of Clinical and Experimental Medicine } \\
\text { Matteoli, Gianluca; KU Leuven, Translational Research in GastroIntestinal } \\
\text { Disorders (TARGID) and Department of Clinical and Experimental Medicine } \\
\text { Deprest, Jan; KU Leuven, Department of Development and Regeneration, } \\
\text { Organ systems cluster, Biomedical Sciences, } \\
\text { Boeckxstaens, Guy; KU Leuven, Translational Research in GastroIntestinal } \\
\text { Disorders (TARGID) and Department of Clinical and Experimental Medicine }\end{array}$ \\
\hline Key Words: & open surgery, laparoscopic surgery, postoperative ileus, inflammation \\
\hline
\end{tabular}

\section{SCHOLARONE ${ }^{\text {M }}$




\section{Absence of intestinal inflammation and postoperative ileus in a mouse model of laparoscopic surgery}

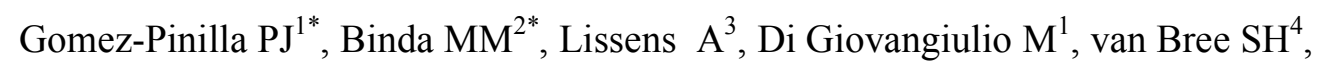
Nemethova $A^{1}$, Stakenborg $\mathrm{N}^{1}$, Farro $\mathrm{G}^{1}$, Bosmans $\mathrm{G}^{1}$, Matteoli $\mathrm{G}^{1}$, Deprest $\mathrm{J}^{5}$, Boeckxstaens $\mathrm{GE}^{1 * *}$

* Contributed equally to this work

** Corresponding author

${ }^{1}$ Translational Research in GastroIntestinal Disorders (TARGID) and Department of Clinical and Experimental Medicine, KU Leuven, Leuven, Belgium.

${ }^{2}$ Laboratory Abdominal Surgical Oncology, Department of Oncology. KU Leuven, Leuven, Belgium.

${ }^{3}$ Centre for Surgical Technologies, KU Leuven, Leuven, Belgium.

${ }^{4}$ Tytgat Institute of Liver and Intestinal Research, Academic Medical Center, Amsterdam, The Netherlands.

${ }^{5}$ Department of Development and Regeneration, Organ systems cluster, Biomedical Sciences, KU Leuven, Leuven, Belgium.

Correspondence to:

Guy E. Boeckxstaens

Department of Clinical and Experimental Medicine and Translational Research Center for Gastrointestinal Disorders (TARGID). University of Leuven

Herestraat 49, O\&N1, bus 701, 3000 Leuven

Tel: +32-16-345750 / +32-16-330237; fax: +32 16330723

E-mail: guy.boeckxstaens@med.kuleuven.be 


\author{
Results of this article were partially presented at the $\mathrm{XXV}^{\text {th }}$ Belgian Week of \\ Gastroenterology, Antwerp, February 28, March 1 - 2, 2013 and at the Digestive Disease \\ Week, May 18-21, 2013, Orlando FL.
}

\begin{abstract}
BACKGROUND: Postoperative ileus (POI) is characterized by impaired gastrointestinal motility resulting from intestinal handling-associated inflammation. The introduction of laparoscopic surgery has dramatically reduced the duration of POI. However, to what extent this results from a reduction in intestinal inflammation remains unclear. The aim of the present study is to compare the degree of intestinal inflammation and gastrointestinal transit following laparoscopic surgery and open abdominal surgery.
\end{abstract}

METHODS: Mice were subjected to laparoscopic surgery or laparotomy alone or in combination with standardized intestinal manipulation of the small bowel (IM). 24 hours after surgery gastrointestinal transit and intestinal inflammation were assessed by the number of myeloperoxidase (MPO) positive cells and the level of cytokine expression. The recovery time and the degree of inflammation were also analyzed in patients subjected to colectomy under open conditions (laparotomy) or laparoscopic conditions.

RESULTS: Mice undergoing IM by laparotomy (open IM), but not by laparoscopy (Lap IM) developed a significant delay in gastrointestinal transit compared to laparotomy or laparoscopy alone. In addition, there was significant intestinal inflammation only after open IM. In line, cytokine levels in peritoneal lavage fluid were lower while recovery time was faster in patients subjected to colectomy under laparoscopic conditions compared to open colectomy.

CONCLUSION: Our data confirm that intestinal inflammation is underlying the delayed gastrointestinal transit observed after open surgery. Most importantly, we demonstrate that 
intestinal inflammation under laparoscopic conditions is significantly lower compared to open surgery, most likely explaining the faster recovery following laparoscopic surgery.

Key words: open surgery, laparoscopic surgery, intestinal manipulation, inflammation, mouse model, colectomy, postoperative ileus

\section{Introduction}

Laparoscopy has become the gold standard for surgical treatment of benign and malignant abdominal disorders. Next to cosmetic and technical benefits such as improved visualization by magnification, laparoscopy is associated with reduced morbidity, reduced pain levels, faster recovery of bowel function, and shorter hospitalization compared to open procedures. ${ }^{1,2}$. Laparoscopy induces less direct trauma because of gentler tissue handling, meticulous hemostasis, constant irrigation, the use of microsurgical instruments and the smaller operative field. ${ }^{3}$ This access method has been associated with less postoperative pain, less systemic immunological depression ${ }^{4,5}$, reduced wound infection, fewer complications, shorter hospital stays and earlier return to normal activities. ${ }^{1,2}$ Even the most radical endoscopic procedures require considerably less recuperation time to normal activities when compared with their open counterpart. ${ }^{3}$ For example in patients with colon cancer, recovery of bowel function is considerably shorter after laparoscopic surgery compared to open colonic resection ${ }^{6}$ as reviewed $i^{7}$. Clinical and animal experimental studies suggest this faster recovery of the bowel motility is mediated by an improved preservation of the host immune defense following laparoscopic procedures ${ }^{4,5}$, but the underlying mechanisms remain unclear. 
Postoperative ileus (POI) is a transient reduction of gut coordinated propulsive motility that occurs following almost each abdominal surgical procedure. Several factors play a role in the occurrence and severity of POI, including the use of anesthetics and opioid analgesics ${ }^{8-10}$ and the opening of the abdominal cavity. The latter activates inhibitory neuronal reflexes involving adrenergic and non-adrenergic pathways that mainly contribute to gut dysmotility in the first 3 hours following surgery. ${ }^{11,12}$ Thereafter, inflammation of the intestinal muscularis externa becomes the main mechanism underlying POI. ${ }^{13-15}$ This inflammatory response consists of activation of resident macrophages located within the muscularis externa by handling of the intestines during surgery. Pro-inflammatory cytokines and chemokines released by activated macrophages, and subsequent expression of adhesion molecules on endothelial cells, will lead to an influx of leukocytes (mainly neutrophils and monocytes) into the muscularis externa. ${ }^{13-15}$ Both incoming monocytes and activated resident macrophages produce nitric oxide and prostaglandins that further compromise the contractile activity of the gut. ${ }^{13-15}$ Hence, from a clinical point of view, this inflammatory phase represents the most important target for treatment. Moreover, since inflammation is one of the main causes leading to POI, we hypothesized that differences in the degree of inflammation may explain the faster recovery following laparoscopic surgery compared to open surgery.

Here, we provide evidence that intestinal manipulation under laparoscopic conditions does not delay gastrointestinal transit and does not induce local intestinal inflammation. This is in line with observations in patients undergoing laparoscopic colectomy, who develop less severe POI and show lower cytokine levels in peritoneal fluid compared to open colectomy. Our findings support the idea that the faster clinical recovery following laparoscopic surgery may be explained by a significant reduction in intestinal inflammatory response to abdominal surgery. 


\title{
Material and Methods
}

\begin{abstract}
Animals
In this study, thirty 10-12 week old C57BL/6JolaHsd female mice (Harlan) were used. Laboratory animals were kept under environmentally controlled conditions $\left(20-22^{\circ} \mathrm{C}, 55 \%\right.$ humidity) with standard mouse chow and water ad libitum. Laparoscopic surgery, laparotomy and intestinal manipulation procedures were approved by the Institutional Review Animal Care Committee.
\end{abstract}

\section{Experimental design for mouse experiments}

Mice were randomly assigned to five different groups: animals not subjected to any surgery $(n=6)$, laparotomy $(n=6)$, laparotomy plus intestinal manipulation (open IM, $n=6$ ), laparoscopy ( $\mathrm{n}=6$ ) and laparoscopy plus intestinal manipulation (Lap IM, n=6). One of the mouse subjected to laparotomy died after surgery by un-known causes. 24 hours after surgery mice were sacrificed and gastrointestinal transit and intestinal inflammation were determined.

\section{Surgical procedure: laparotomy and laparoscopic surgery}

Mice were anesthetized by intraperitoneal (i.p.) injection of a mixture of Ketamine (Ketalar $100 \mathrm{mg} / \mathrm{kg}$; Pfizer) and Xylazine (Rompun $10 \mathrm{mg} / \mathrm{kg}$; Bayer). Anesthetized mice were shaved at the level of the abdomen and laparotomy or laparoscopic surgery was performed according to the study group. For open surgery, a $25 \mathrm{~mm}$ middle abdominal incision was done and the peritoneum was opened over the linea alba. Thereafter, the small bowel was eventrated using two cotton swabs (Fig. 1A) as previously described. ${ }^{16}$

For laparoscopic surgery, animals were intubated with a 20-gauge catheter and mechanically ventilated (Mouse Ventilator MiniVent, type 845, Hugo Sachs Elektronik-Harvard Apparatus $\mathrm{GmbH}$, March-Hugstetten, Germany) using humidified room air with a tidal volume of 250 
$\mu \mathrm{L}$ at 160 strokes. An inguinal midline incision was made and a $2-\mathrm{mm}$ endoscope with a 3.3mm external sheath for insufflation (Karl Storz, Tuttlingen, Germany) was introduced into the abdominal cavity (Fig. 1B-C) as previously published. ${ }^{17}$ The incision around the entry site was sealed gas tight with a purse string to avoid leakage. A pneumoperitoneum (pressure) was created with the Thermoflator Plus (Karl Storz) using pure carbon dioxide. After induction of the pneumoperitoneum, two 14-gauge catheters were inserted under laparoscopic vision to perform the intestinal manipulation as explained below.

\section{Intestinal manipulation}

The small intestine was manipulated (IM) by the same surgeon (MMB) to avoid variability. For IM, the small intestine was manipulated using a $1.5 \mathrm{~mm}$ grasper and the plastic tip of a 14 GA trocar (Fig. 1A-B). IM was performed from the caecum to the distal duodenum and contact or stretching of the stomach or colon was strictly avoided. Manipulation took between 3-5 and 6-8 minutes for open and laparoscopic surgery, respectively.

After the surgical procedure, the abdomen was closed by a continuous two-layer suture (Mersilene, 6-0 silk) in animals subjected to laparotomy while in animals subjected laparoscopy three small abdominal incision points were closed with suture (Mersilene, 6-0 silk) too. Surgery was performed under sterile conditions and body temperature was kept around $37^{\circ} \mathrm{C}$ during the procedure. After closure, mice were allowed to recover for 3 hours in a heated pad $\left(37^{\circ} \mathrm{C}\right)$ recovery cage without administration of anti-inflammatory or analgesic agents as these can alter gastrointestinal motility and the postoperative process. Thereafter, food and water were provided ad libitum.

\section{Gastrointestinal transit measurement}

To assess gastrointestinal transit, 24 hours after surgery mice were gavaged with a liquid nonabsorbable fluorescein isothiocyanate-labeled dextran (FITC-dextran, 70,000 Da; Invitrogen). 
After 90 minutes, animals were sacrificed and the contents of stomach, small bowel (divided into 10 segments of equal length), caecum, and colon (3 segments of equal length) were collected. The amount of FITC in each bowel segment was quantified using a spectrofluorimeter (Ascent, Labsystem Inc). The distribution of the fluorescent dextran along the gastrointestinal tract was determined by calculating the geometric center (GC) value for quantitative comparisons among experimental groups. ${ }^{15,16,18}$

GC: $\Sigma$ (percent of total fluorescent signal in each segment $\mathrm{x}$ the segment number)/100

\section{Intestinal inflammation analysis}

\section{Myeloperoxidase staining}

A 2-cm long fragment of the jejunum was fixed with $100 \%$ ethanol for 10 minutes. Next, the mucosa and submucosa were removed and the remaining full-thickness sheets of muscularis externa were stained with Hanker Yates reagent (Sigma-Aldrich) for 10 minutes. ${ }^{19}$ Myeloperoxidase (MPO) positive cells were visualized with a microscope (BX 41 Olympus) connected to a camera (XM10 Olympus) and $\mathrm{Cell}^{\wedge} \mathrm{F}$ software was used for sampling the tissues. The number of MPO-positive cells in 10 randomly chosen representative high-power magnification fields (taken with the 10X objective, $668.4 \mu \mathrm{m} \times 891.2 \mu \mathrm{m}$ ) was counted by a blinded investigator.

\section{Inflammatory gene expression}

Total RNA was extracted from the muscularis externa of the jejunum 24 hours after surgery to analyze the mRNA expression level of the following inflammation related genes: interleukin 6 (ilo), interleukin 1 alpha $(i l 1 a)$ and interleukin 1 beta $(i l l b)$. Tissues were homogenized by the TissueLyser II homogenizer (Qiagen). RNA extraction was performed using RNeasy Mini Kit (Qiagen) following the manufacturer's instructions. Total RNA was 
transcribed into complementary cDNA by qScript cDNA SuperMix (Quanta Biosciences) according to the manufacturer's instructions. Quantitative real-time transcription polymerase chain reactions (RT-PCR) were performed with the LightCycler 480 SYBR Green I Master (Roche) on the Light Cycler 480, (Roche). Results were quantified using the 2- $\Delta \Delta \mathrm{CT}$ method. The expression levels of the genes of interest were normalized to the expression levels of the reference gene rp132. PCR experiments were performed in triplicate. Primer sequences used are listed in table 1.

$\underline{\text { Table 1. Primer sequences used for qRT-PCR }}$

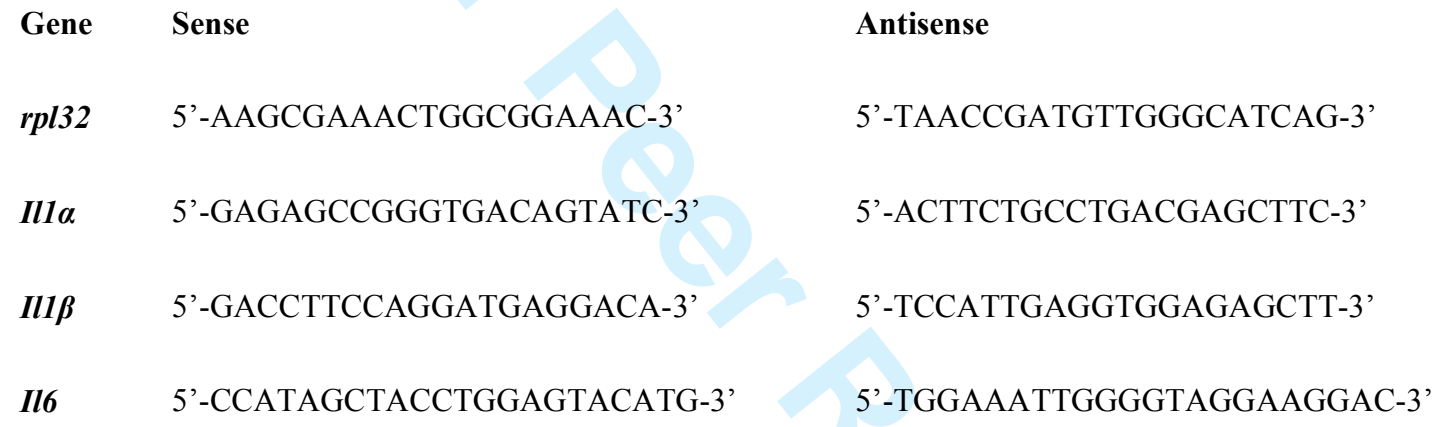

\section{Clinical study}

\section{Patients}

Patients were invited to participate when undergoing elective segmental colectomy for colonic cancer without evidence of metastatic disease. Informed consent was signed by all the patients included in the study. Patients were subjected to segmental colectomy under open conditions (laparotomy, $n=22)$ or laparoscopic conditions $(n=27)$. The study was conducted in the Academic Medical Center (Amsterdam, The Netherlands) in accordance with the principles of the Declaration of Helsinki. The protocol was approved by the Medical Ethics Review Board of the Academic Medical Center in Amsterdam, The Netherlands (National Trial Register, number NTR1884). 


\section{Analysis of peritoneal lavage fluid}

Peritoneal lavage fluid samples were collected from patients undergoing a colectomy under open or laparoscopic conditions. Peritoneal lavages samples were collected at the end of the surgical procedure before closing the abdominal cavity or the small incisions. The abdominal lavages were performed using $100 \mathrm{~mL}$ of warm $\left(42^{\circ} \mathrm{C}\right)$ sterile $0.9 \% \mathrm{NaCl}$ solution, which was sprinkled gently onto the small intestine and its mesentery. After approximately 30 seconds, peritoneal fluid (between 20 and $40 \mathrm{~mL}$ ) was collected using a 22 French Foley catheter (Bard Limited, West Sussex, UK) connected to a $50 \mathrm{~mL}$ catheter tip syringe.

Peritoneal levels of I16, Il1 $\alpha, \mathrm{Il} 1 \beta, \mathrm{Il} 8, \mathrm{I} 112 \mathrm{p} 70$ and TNF $\alpha$ were determined using Cytometric Bead Array (CBA) kits for human (for TNF $\alpha$, IL12p70 and IL1ß the enhanced sensitivity flex set kits were used) according to the manufacturer's instructions (BD Biosciences). Flow cytometric analysis was performed using a FACS Array flow cytometer (BD Biosciences). CBA results were analyzed using the FCAP ArrayTM software (BD Biosciences).

\section{Clinical end points}

To track the postoperative time to tolerance of solid food, patients were assisted (by a trial nurse and/or a research physician) to complete a self-assessment sheet daily until hospital discharge. Time to tolerance of solid food (TSF) was defined as the first time the subject was able to eat solid food (any food that required chewing) without vomiting or experiencing significant nausea within 4 hours following the meal, or without having to revert to enteral fluids. All patients were discharged according to the same predefined discharge criteria and time to hospital discharge (HD) was recorded.

\section{Statistical analysis}


All mouse data were statistically analyzed by one-way analysis of variance followed by Bonferroni's multiple comparison test. In the clinical study, normally distributed data were analyzed using parametric tests. Data that were not normally distributed were subjected to non-parametric test (Mann Whitney test). Probability level of $\mathrm{p}<0.05$ was considered statistically significant and results are shown as mean \pm SEM. Graph Pad Prism V.5.01 software was used to perform statistical analysis and create graphs.

\section{Results}

Intestinal manipulation under laparoscopic conditions does not induce a delay in gastrointestinal transit

Geometric center (GC) values, as index of gastrointestinal transit, from animals subjected to laparotomy $(\mathrm{GC} ; 10.06 \pm 0.30, \mathrm{n}=5)$ and laparoscopy $(\mathrm{GC} ; 9.92 \pm 0.32, \mathrm{n}=6)$ were similar to those found in control mice not subjected to any surgery $(\mathrm{GC} ; 10.33 \pm 0.26, \mathrm{n}=6$, Fig. $2 \mathrm{~A}, \mathrm{~ns}$, one -way ANOVA). In contrast, open IM of the small intestine resulted in a delay of the intestinal transit (Fig. 2B) ( $<<0.05$, one-way ANOVA). Interestingly, lap IM did not result in a delay of the gastrointestinal transit compared with laparoscopy alone (Fig. 2C) (ns, one-way ANOVA). Notably, there was a significant reduction in GC values from animals subjected to open IM when compared to Lap IM group (Fig. 2D, p<0.05, one-way ANOVA).

\section{Intestinal manipulation under laparoscopic conditions does not lead to intestinal inflammation}

As shown in figures 3 and 4, laparotomy and laparoscopy alone did not induce intestinal inflammation with similar numbers of myeloperoxidase (MPO) positive cells in the intestinal muscularis $(2.6 \pm 2.1$ and 10.1 $\pm 8.8 \mathrm{MPO}$-positive cells/field for laparotomy and laparoscopy group respectively vs $1.6 \pm 1.8 \mathrm{MPO}$-positive cells/field in control mice not subjected to surgery, ns, one-way ANOVA, Fig. 3). In addition, no differences were found between il6, 
$i l l a$ and ill $b$ mRNA expression (ns, one-way ANOVA, Fig. 4). However, open IM resulted in a significant increase in MPO positive cells recruited in the muscularis externa. In line, open IM significantly increases the expression levels of $i l 6, i l l a$ and $i l l b$ compared to laparotomy alone, laparoscopy alone and Lap IM (Fig. 3-4). Lap IM failed to evoke an inflammatory response compared to laparoscopy alone.

\section{Laparoscopic colectomy leads to less severe POI and reduced inflammation compared to conventional colectomy in humans}

The time until tolerance to solid food (TSF) and until hospital discharge (HD) was significantly reduced in patients subjected to laparoscopic colectomy compared with patients subjected to open colectomy (TSF; $p=0.0008$ and HD; $p=0.0001$, non-parametric test (Mann Whitney test), Fig. 5).

In parallel, the levels of pro-inflammatory cytokines (I16, Il1 $\alpha, \mathrm{Il} 8, \mathrm{Il12p} 70$ and TNF $\alpha$ ) in peritoneal lavages were higher after open intestinal surgery compared to those in patients who underwent a laparoscopic colectomy (Il6; $\mathrm{p}=0.026, \mathrm{Il} 1 \alpha ; \mathrm{p}=0.019, \mathrm{Il} ; \mathrm{p}=0.001, \mathrm{Il} 12 \mathrm{p} 70 ; \mathrm{p}=$ 0.013 and TNF $\alpha p=0.001$, non-parametric test (Mann Whitney test), Fig. 6) while for Il1 $\beta$ there was no significant difference between the two groups.

\section{Discussion}

Minimally invasive laparoscopic surgery is one of the most important advances in modern surgical care. Laparoscopy is safer compared to open surgery, and effectively reduces surgery-associated trauma and morbidity leading to faster recovery from surgery. The underlying mechanisms, however, remain unclear.

In the late 1990s, intestinal inflammation was demonstrated to be the main pathophysiological mechanism underlying postoperative ileus (POI). Manipulation of the intestine during surgery 
triggers the activation of intestinal resident macrophages and consequently the influx of leucocytes to the manipulated intestine starting approximately $3-4 \mathrm{~h}$ after surgery. ${ }^{13-15,18}$ These inflammatory cells release NO and prostaglandins impairing smooth muscle contractility thereby mediating POI. ${ }^{20,21}$ Indeed, anti-inflammatory strategies to prevent POI such as activation of the cholinergic anti-inflammatory pathway ${ }^{22,23}$, NSAIDs $^{24}$ and drugs that target mast cells ${ }^{25,26}$, resident macrophages ${ }^{18,25}$ and neutrophils ${ }^{15,27}$ have been shown to be effective in reducing POI. ${ }^{7}$

To evaluate the hypothesis that faster recovery following laparoscopy is associated with less intestinal inflammation, we made use of a laparoscopic and an open surgery mouse model. The animal model of intestinal manipulation (IM) of the small bowel by means of two cotton applicators is currently widely used to induce POI in mice and rat as it mimics the abdominal handling of the gut in patients undergoing abdominal surgery. ${ }^{13,14,28}$ In the current study, we adapted this technique to a model of laparoscopic surgery to compare the effects of the two different surgical techniques on intestinal inflammation and recovery of gastrointestinal transit. In contrast to open IM, no delay in intestinal transit or intestinal inflammation was observed with laparoscopic IM. In line, in patients subjected to segmental colectomy, the levels of pro-inflammatory cytokines detected in the peritoneal lavage fluid were significantly lower under laparoscopic conditions, a finding that was associated with a shorter POI compared to patients undergoing open surgery. Based on these findings, we conclude that faster recovery after laparoscopic surgery may result from a reduced inflammatory response to surgical handling of the intestine.

Although we did not measure tissue damage in the present study, one can speculate that laparoscopic surgery is less traumatic than open surgery. The intestine is handled with more care during laparoscopic surgery, and in addition, the intestine is exposed to a completely different environment in the two types of surgery. Under normal physiological conditions, the 
partial pressure of oxygen $\left(\mathrm{ppO}_{2}\right)$ in the tissues is around $23 \mathrm{~mm} \mathrm{Hg}$. During standard laparoscopy, pure $\mathrm{CO}_{2}$ is insufflated into the peritoneal cavity to induce a pneumoperitoneum. Hence, the abdominal cavity and specially the mesothelial layer covering the bowel serosa are exposed to a hypoxic environment, hypothermia and barotrauma. During open surgery, the abdominal cavity and the intestines are exposed to air composed of $20.9 \%$ oxygen (160 mm Hg). This hyperoxic environment induces the production of reactive oxygen species (ROS) and oxidative stress. ${ }^{29}$ Moreover, we previously showed that open surgery performed in a dry environment $(0 \%$ relative humidity) induces desiccation of the tissue, associated with increased mortality (unpublished results). We can, therefore, hypothesize that during open surgery both the hyperoxic and dry environment will induce more tissue damage, a key player in triggering sterile inflammation and most likely contributing to the activation of the resident macrophages in the intestinal muscularis. Further experiments are however required to confirm this hypothesis.

Our findings are consistent with previous results showing that following laparoscopic cholecystectomy in dogs, the reduction in intestinal motility is less in comparison with open cholecystectomy. ${ }^{30,31,32}$ According to the authors, this difference resulted from the lower extent of abdominal trauma associated with the laparoscopic technique. Other factors that may contribute to faster recovery following laparoscopic surgery are a reduced stress response (lower levels of epinephrine and norepinephrine) ${ }^{33}$ and a better preserved immune response allowing more effective elimination of pathogens (higher level of HLA-DR) ${ }^{34}$ compared to conventional open surgery. Here we provide evidence in mice and humans that faster postoperative recovery of gut motility coincides with a reduction in the inflammatory response to intestinal handling.

In conclusion, we provide evidence in mice that manipulation of the intestine triggers an inflammatory response and reduces intestinal transit under open but not under laparoscopic 
conditions. Similarly, the levels of pro-inflammatory cytokines are lower in patients undergoing laparoscopic compared to open segmental colectomy. Based on these findings, we propose that the reduced inflammatory response of the muscularis externa observed after laparoscopic surgery is a new mechanism that contributes to the faster clinical recovery compared to open surgery. We hypothesize that exposure of the abdominal organs to a nonphysiologic environment during open surgery (high concentration of oxygen and dry air) may contribute to the increased intestinal inflammation and more severe impairment in gastrointestinal transit observed following these procedures.

\section{Acknowledgements}

Karl Storz is kindly thanked for providing the laparoscopic material for the laparoscopic mouse model. We thank the department of anesthesiology, W. Bemelman, M. van Berge Henegouwen, D.J. Gouma and the GI surgery fellows at the department of surgery of the Academic Medical Center in Amsterdam for their support with collecting the patient abdominal lavage samples. Lisbeth Vercruysse is thanked for helping with the design of mouse model figure.

Supported by grants from Research Foundation - Flanders (FWO) (Odysseus and Hercules program to G.E.B.), by a FWO postdoctoral research fellowship (to G.M. and P.J.G-P) and by FWO PhD fellowship (to M.DG). JD is a beneficent of a fundamental clinical research grant of the Fonds Wetenschappelijk Onderzoek Vlaanderen (1.8.012.07).

\section{Author contributions}

P.J. G-P, M.M.B, A.L, M.DG, S.H. vB, A.N, N.S, G.F, G.B and G.M performed the experiments and were responsible for acquisition and analysis of data and drafting of the manuscript. P.J. G-P, M.M.B, A.L, J.D and G.E.B handled study concept and design, analysis 
and interpretation of data, and critical revision of the manuscript for important intellectual content. J.D and G.E.B obtained funding.

\section{Conflict of interests}

No conflict of interest exists that could be perceived to bias the work.

\section{References list}

1. Sajid MS, Bokhari SA, Mallick AS, Cheek E, Baig MK. Laparoscopic versus open repair of incisional/ventral hernia: a meta-analysis. American journal of surgery 2009; 197: 64-72.

2. Chung RS, Rowland DY, Li P, Diaz J. A meta-analysis of randomized controlled trials of laparoscopic versus conventional appendectomy. American journal of surgery 1999; 177: 250-256.

3. Azziz R, Steinkampf MP, Murphy A. Postoperative recuperation: relation to the extent of endoscopic surgery. Fertility and sterility 1989; 51: 1061-1064.

4. Collet D, Vitale GC, Reynolds M, Klar E, Cheadle WG. Peritoneal host defenses are less impaired by laparoscopy than by open operation. Surgical endoscopy 1995; 9: 10591064.

5. Allendorf JD, Bessler M, Whelan RL, et al. Postoperative immune function varies inversely with the degree of surgical trauma in a murine model. Surgical endoscopy 1997; 11: 427-430.

6. Ohtani H, Tamamori Y, Arimoto Y, Nishiguchi Y, Maeda K, Hirakawa K. A metaanalysis of the short- and long-term results of randomized controlled trials that compared laparoscopy-assisted and open colectomy for colon cancer. Journal of Cancer 2012; 3: 49-57. 
7. van Bree SH, Nemethova A, Cailotto C, Gomez-Pinilla PJ, Matteoli G, Boeckxstaens GE. New therapeutic strategies for postoperative ileus. Nature reviews Gastroenterology \& hepatology 2012; 9: 675-683.

8. Bueno L, Ferre JP, Ruckebusch Y. Effects of anesthesia and surgical procedures on intestinal myoelectric activity in rats. The American journal of digestive diseases 1978; 23: 690-695.

9. Bauer AJ, Sarr MG, Szurszewski JH. Opioids inhibit neuromuscular transmission in circular muscle of human and baboon jejunum. Gastroenterology 1991; 101: 970-976.

10. Bauer AJ, Szurszewski JH. Effect of opioid peptides on circular muscle of canine duodenum. The Journal of physiology 1991; 434: 409-422.

11. Holzer P, Lippe IT, Amann R. Participation of capsaicin-sensitive afferent neurons in gastric motor inhibition caused by laparotomy and intraperitoneal acid. Neuroscience 1992; 48: $715-722$.

12. Boeckxstaens GE, Hirsch DP, Kodde A, et al. Activation of an adrenergic and vagally-mediated NANC pathway in surgery-induced fundic relaxation in the rat. Neurogastroenterology and motility : the official journal of the European Gastrointestinal Motility Society 1999; 11: 467-474.

13. Kalff JC, Carlos TM, Schraut WH, Billiar TR, Simmons RL, Bauer AJ. Surgically induced leukocytic infiltrates within the rat intestinal muscularis mediate postoperative ileus. Gastroenterology 1999; 117: 378-387.

14. Kalff JC, Buchholz BM, Eskandari MK, et al. Biphasic response to gut manipulation and temporal correlation of cellular infiltrates and muscle dysfunction in rat. Surgery 1999; 126: 498-509. 
15. de Jonge WJ, van den Wijngaard RM, The FO, et al. Postoperative ileus is maintained by intestinal immune infiltrates that activate inhibitory neural pathways in mice. Gastroenterology 2003; 125: 1137-1147.

16. van Bree SH, Nemethova A, van Bovenkamp FS, et al. Novel method for studying postoperative ileus in mice. International journal of physiology, pathophysiology and pharmacology 2012; 4: 219-227.

17. Binda MM, Molinas CR, Hansen P, Koninckx PR. Effect of desiccation and temperature during laparoscopy on adhesion formation in mice. Fertility and sterility 2006; 86: 166-175.

18. Wehner S, Behrendt FF, Lyutenski BN, et al. Inhibition of macrophage function prevents intestinal inflammation and postoperative ileus in rodents. Gut 2007; 56: 176-185.

19. Kalff JC, Schwarz NT, Walgenbach KJ, Schraut WH, Bauer AJ. Leukocytes of the intestinal muscularis: their phenotype and isolation. Journal of leukocyte biology 1998; 63: 683-691.

20. Kalff JC, Schraut WH, Billiar TR, Simmons RL, Bauer AJ. Role of inducible nitric oxide synthase in postoperative intestinal smooth muscle dysfunction in rodents. Gastroenterology 2000; 118: 316-327.

21. Kreiss C, Birder LA, Kiss S, VanBibber MM, Bauer AJ. COX-2 dependent inflammation increases spinal Fos expression during rodent postoperative ileus. Gut 2003; 52: 527-534.

22. de Jonge WJ, van der Zanden EP, The FO, et al. Stimulation of the vagus nerve attenuates macrophage activation by activating the Jak2-STAT3 signaling pathway. Nature immunology 2005; 6: 844-851. 
23. Matteoli G, Gomez-Pinilla PJ, Nemethova A, et al. A distinct vagal anti-inflammatory pathway modulates intestinal muscularis resident macrophages independent of the spleen. Gut 2013.

24. Schwarz NT, Kalff JC, Turler A, et al. Prostanoid production via COX-2 as a causative mechanism of rodent postoperative ileus. Gastroenterology 2001; 121: 1354-1371.

25. van Bree SH, Gomez-Pinilla PJ, van de Bovenkamp FS, et al. Inhibition of spleen tyrosine kinase as treatment of postoperative ileus. Gut 2013; 62: 1581-1590.

26. The FO, Buist MR, Lei A, et al. The role of mast cell stabilization in treatment of postoperative ileus: a pilot study. The American journal of gastroenterology 2009; 104: 22572266.

27. The FO, de Jonge WJ, Bennink RJ, van den Wijngaard RM, Boeckxstaens GE. The ICAM-1 antisense oligonucleotide ISIS-3082 prevents the development of postoperative ileus in mice. British journal of pharmacology 2005; 146: 252-258.

28. Kalff JC, Schraut WH, Simmons RL, Bauer AJ. Surgical manipulation of the gut elicits an intestinal muscularis inflammatory response resulting in postsurgical ileus. Annals of surgery 1998; 228: 652-663.

29. Binda MM, Molinas CR, Koninckx PR. Reactive oxygen species and adhesion formation: clinical implications in adhesion prevention. Human reproduction 2003; 18: 2503 2507.

30. Tittel A, Schippers E, Grablowitz V, et al. Intraabdominal humidity and electromyographic activity of the gastrointestinal tract. Laparoscopy versus laparotomy. Surgical endoscopy 1995; 9: 786-790.

31. Tittel A, Schippers E, Anurov M, Titkova S, Ottinger A, Schumpelick V. [Minor abdominal trauma by laparoscopic surgery? Comparison of adhesion formation and intestinal 
motility after laparoscopic and conventional operations in the dog]. Zentralblatt fur Chirurgie $1996 ; 121: 329-334$.

32. Schippers E, Ottinger AP, Anurov M, Polivoda M, Schumpelick V. [Intestinal motility after laparoscopic vs conventional cholecystectomy. An animal experiment study and clinical observation]. Langenbecks Archiv fur Chirurgie 1992; 377: 14-18.

33. Friedrich M, Rixecker D, Friedrich G. Evaluation of stress-related hormones after surgery. Clinical and experimental obstetrics \& gynecology 1999; 26: 71-75.

34. Schietroma M, Carlei F, Lezoche E, et al. Evaluation of immune response in patients after open or laparoscopic cholecystectomy. Hepato-gastroenterology 2001; 48: 642-646.

\section{Figures legend}

Figure 1. Methodology used to perform intestinal manipulation in mice. (A) Image shows manual manipulation of the small intestine using a $1.5 \mathrm{~mm}$ grasper and the plastic tip of a 14 GA trocar under open conditions. (B) Image shows manual manipulation of the small intestine using a small grasper and the plastic tip of a 14 GA trocar under laparoscopic conditions. (C) Scheme of the laparoscopic setup used to perform intestinal manipulation under laparoscopic conditions.

Figure 2. Intestinal manipulation under laparoscopic conditions does not delay gastrointestinal transit. (A) Dextran transit through the intestinal segments from one C57BL/6JolaHsd female mouse that was not subjected to any type of surgery or anesthesia treatment. (B) Dextran transit through the intestinal segments from mice subjected to laparotomy alone (black) and laparotomy plus intestinal manipulation (IM, grey). Note that IM delayed the passage of the dextran through the gut. (C) Dextran transit through the intestinal segments from mice 
subjected to laparoscopy alone (black) and laparoscopy plus intestinal manipulation (IM, grey). Note that there was no impact of IM under laparoscopic conditions. (D) Geometric center (GC) values of dextran distribution for the four groups of animals. IM plus laparotomy group presents a reduction in GC values compared to IM plus laparoscopy. Data in B and C show the mean for each segment from 5 or 6 mice per group. Histogram shows dots distribution and mean \pm SEM of the GC value for each group. $* P<0.05$ compared with laparoscopy plus IM group (one-way ANOVA followed by Bonferroni post-hoc).

Figure 3. Intestinal manipulation under laparoscopic conditions does not induce MPO positive cell influx to the muscularis. (A) Representative images of MPO-positive cells recruited into the muscularis externa of the murine jejunum 24 hours after laparotomy, or laparoscopy alone, or in combination with IM. Scale bar $100 \mu \mathrm{m}$. (B) Histogram shows dots distribution and mean \pm SEM of the MPO-positive cells/field for the four experimental groups of animals. Only laparotomy plus IM lead to a significantly high MPO-positive cells influx to the muscularis externa. ${ }^{*} P<0.01$ compared with laparotomy plus IM group (one-way ANOVA followed by Bonferroni post-hoc).

Figure 4. Absence of intestinal inflammation in IM performed under laparoscopic conditions. il6 (A), illa (B) and illb (C) mRNA expression in the muscularis externa of the jejunum 24 hours after surgical procedures for the five experimental groups of animals. Interestingly there was a significant increase in expression of inflammatory markers exclusively in the group subjected to laparotomy plus IM. Data are expressed in respect to the housekeeping gene $r p l 32$. Histograms show dots distribution and mean \pm SEM for every group of animals. ${ }^{*} P<$ $0.05, * * P<0.01$ and $* * * P<0.001$ compared with laparotomy plus IM group (one-way ANOVA followed by Bonferroni post-hoc). 
Figure 5. Laparoscopic conditions reduce the time to recovery of gut motility and the hospitalization after colectomy. Time until tolerance to solid food (A) and hospital discharge (B) in patient subjected to open colectomies (black) and laparoscopic colectomies (grey). Duration of GI recovery is longer in the open colectomy group. Histograms show dots distribution and mean \pm SEM in days for both groups of patients. $* * * P<0.01$ compared with open colectomy group (non-parametric test followed by Mann Whitney test).

Figure 6. Colectomy under laparoscopic conditions leads to less inflammation compared to open colectomy. Peritoneal content of pro-inflammatory cytokines were analyzed after colectomies under open and laparoscopic conditions. Peritoneal level of Il6 (A), Il1 $\alpha$ (B), Il1 $\beta$ (C), Il8 (D), Il 12p70 (E) and TNF $\alpha$ (F) were substantially reduced after laparoscopiccompared to open procedures. Note that there was no significant reduction for Il1 $\beta$ (C). Histograms show dots distribution and mean $\pm \mathrm{SEM}$ in $\mathrm{pg} / \mathrm{ml}$ or $\mathrm{fg} / \mathrm{ml}$ for both groups of patients. ${ }^{*} P<0.05$ and ${ }^{* *} P<0.01$ compared with open colectomy group (non-parametric test followed by Mann Whitney test). 


\section{Figure 1}
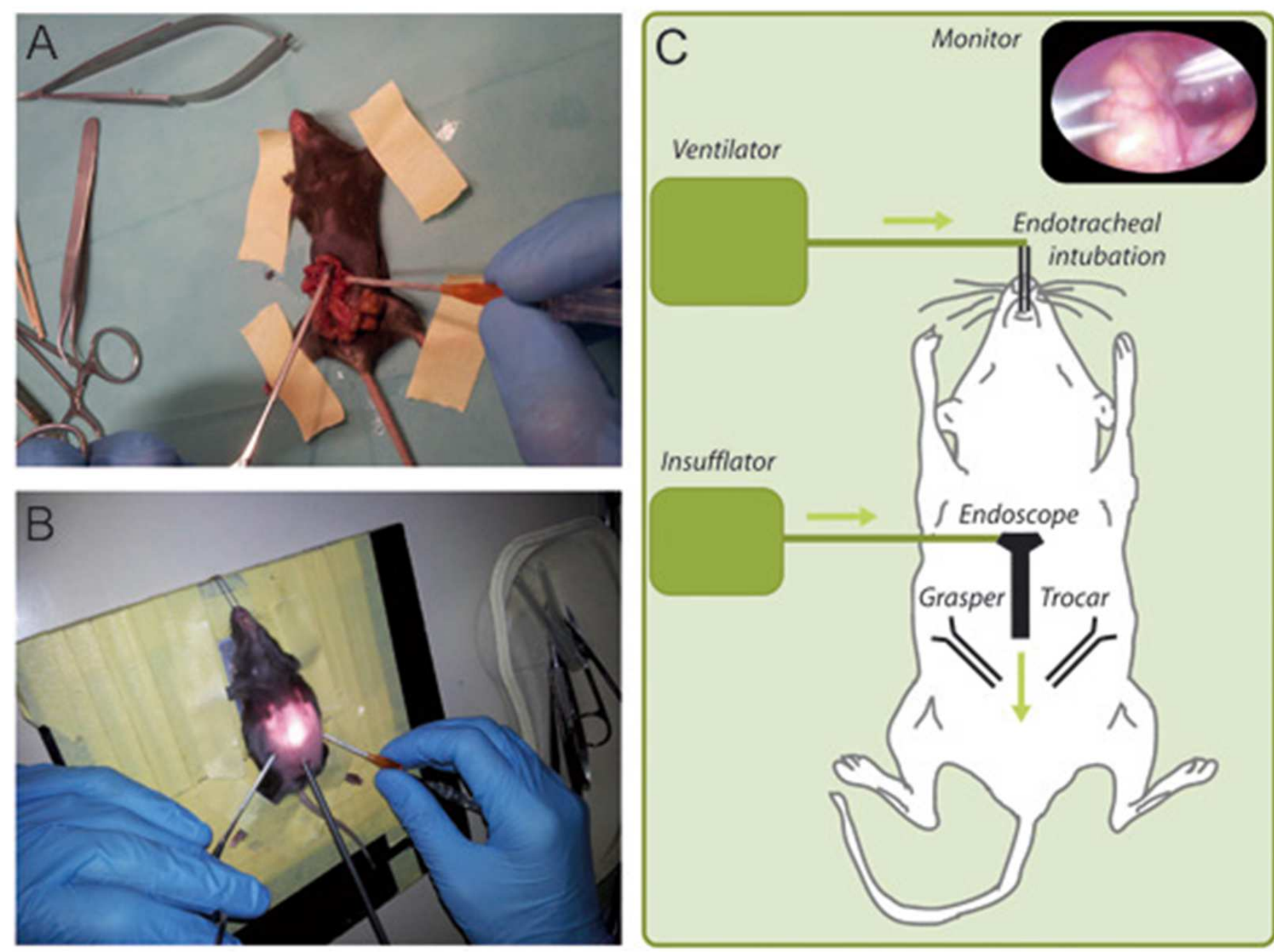


\section{Figure 2}

A)

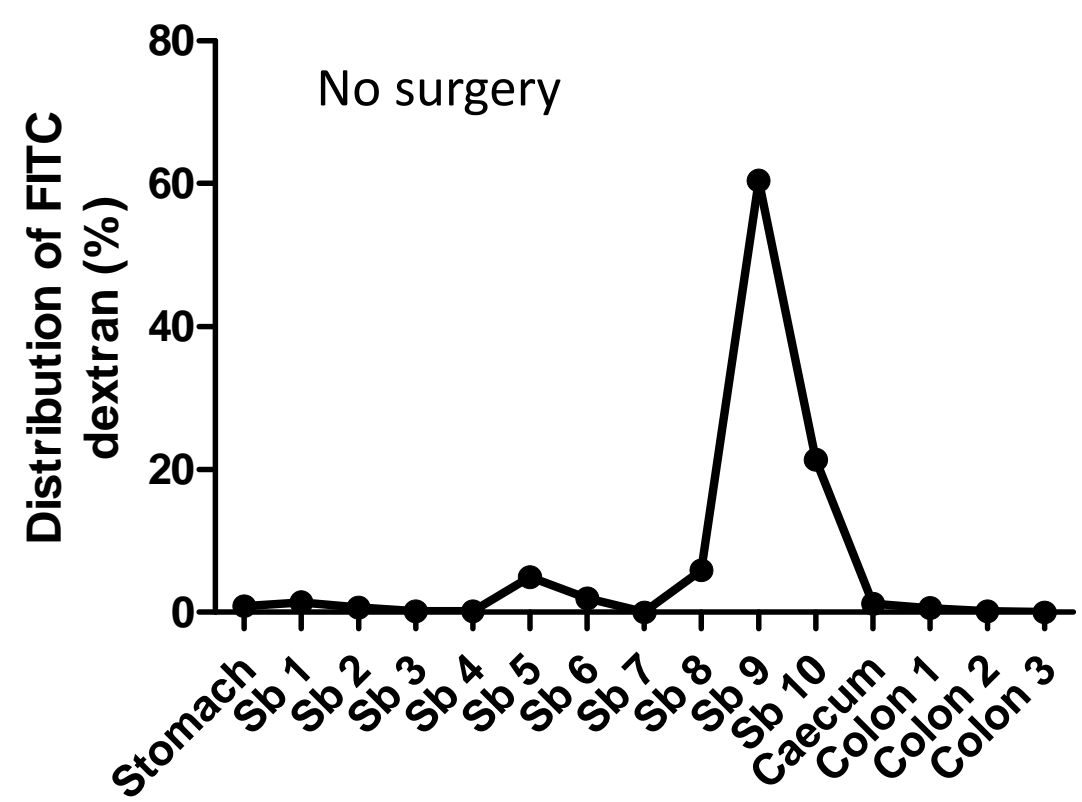

C)

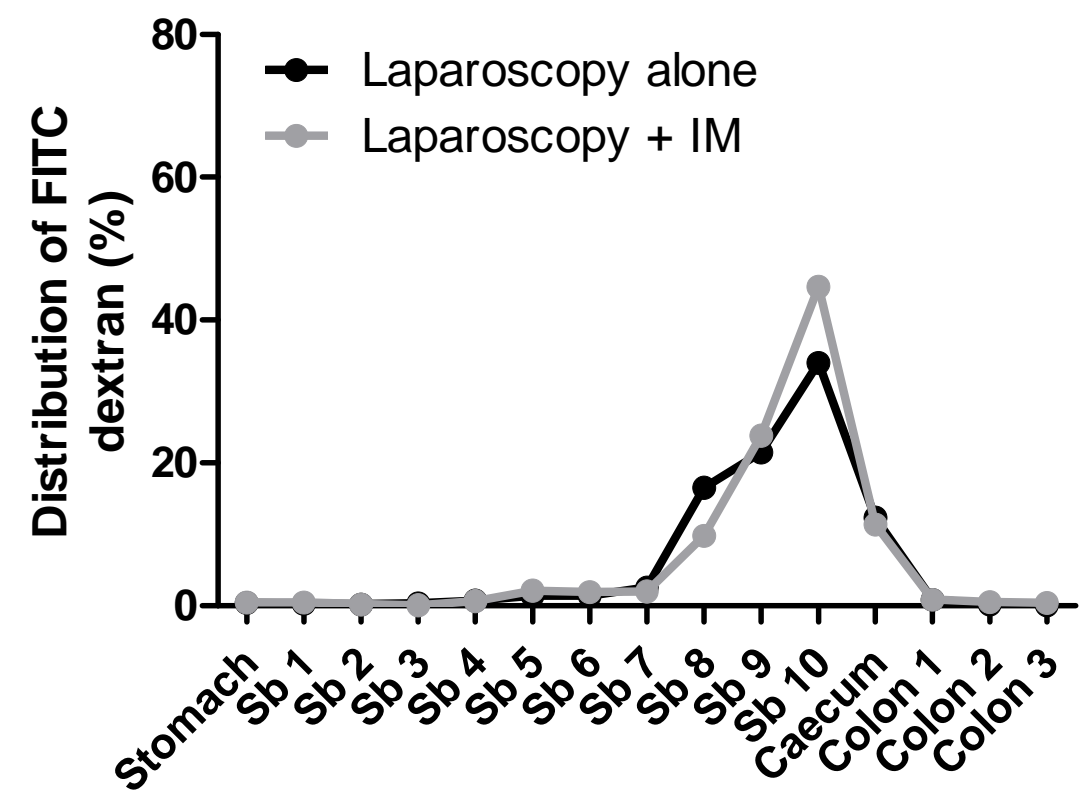

B)

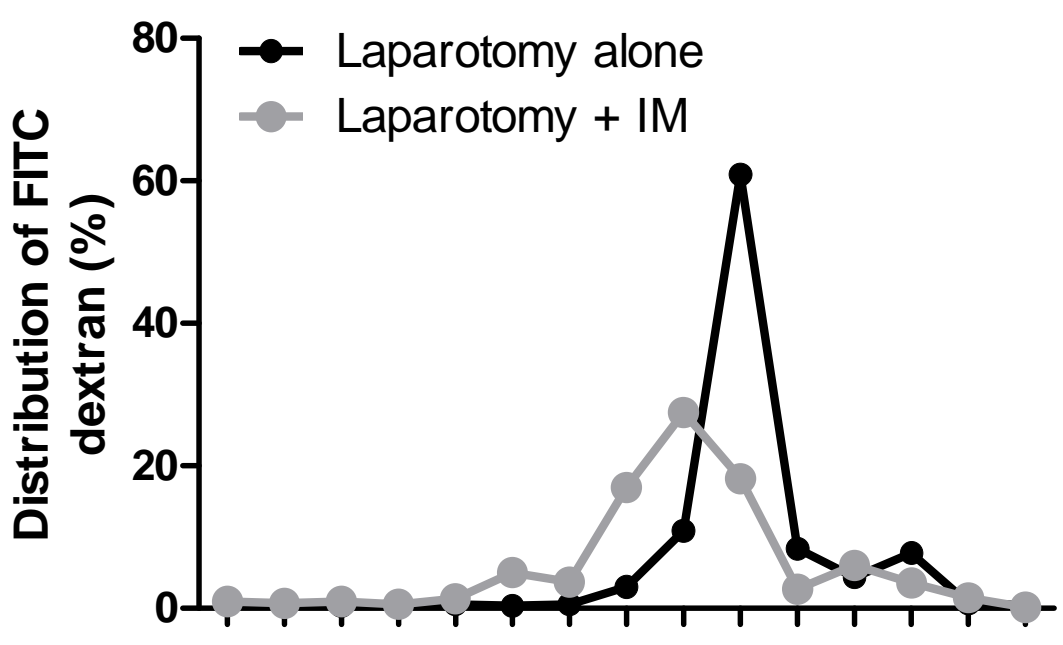

D)
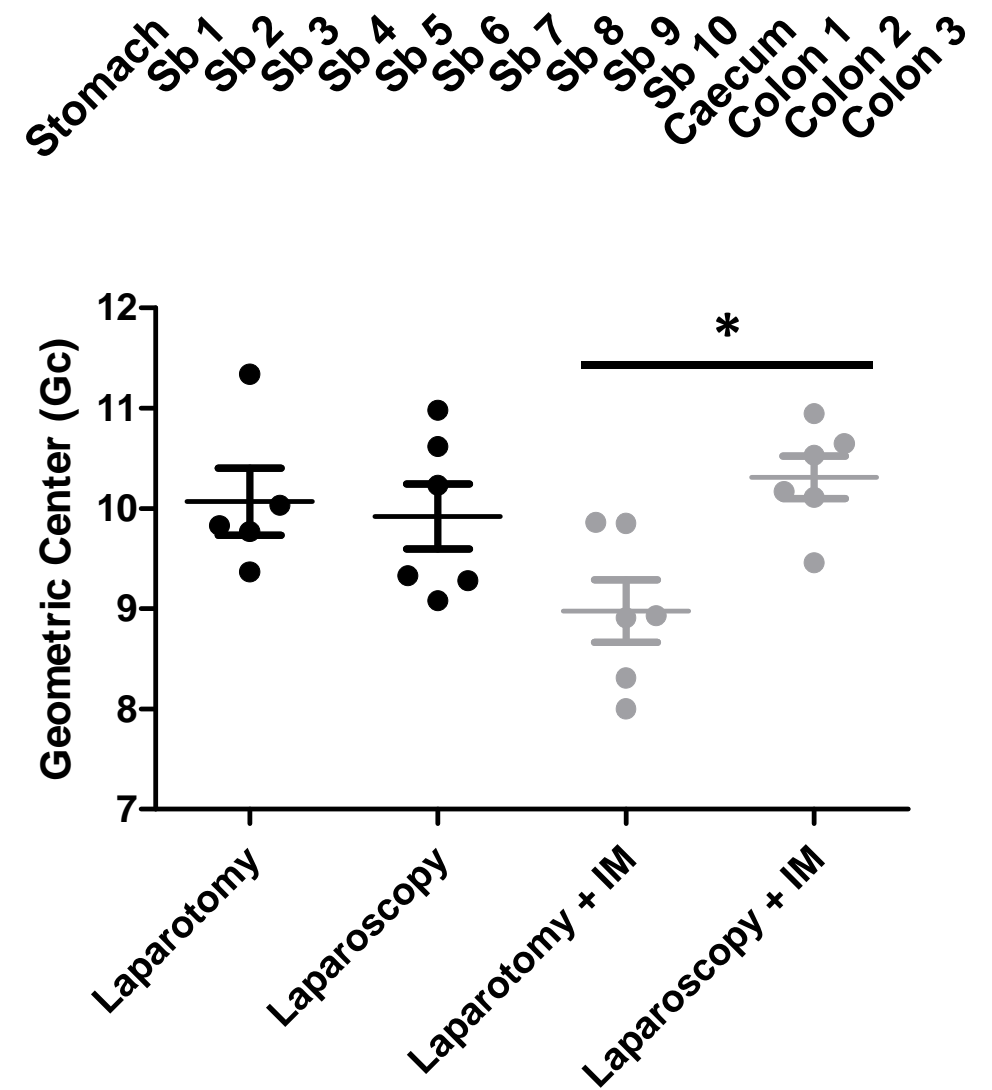


\section{Figure 3}

A)

B)

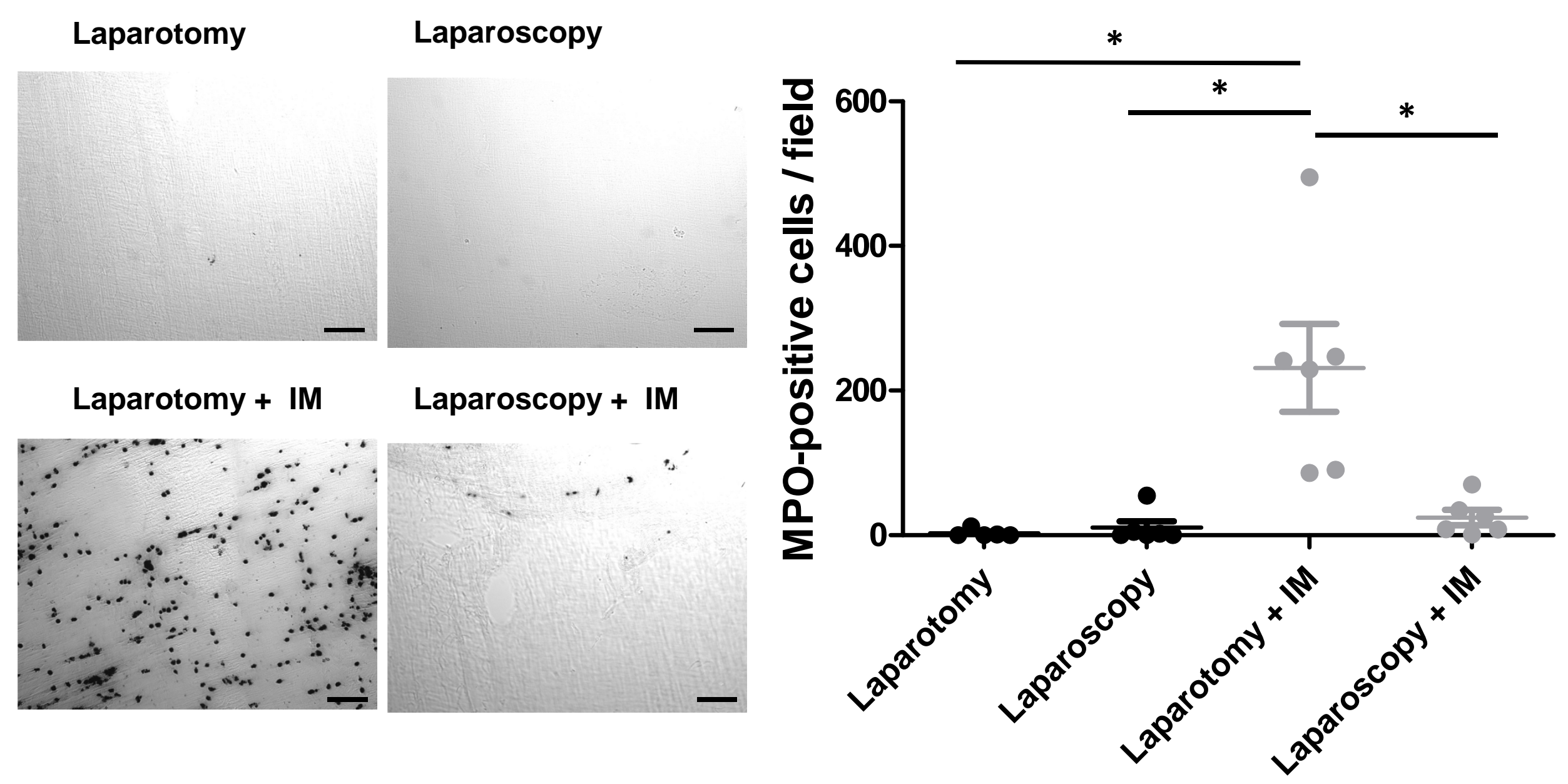




\section{Figure 4}

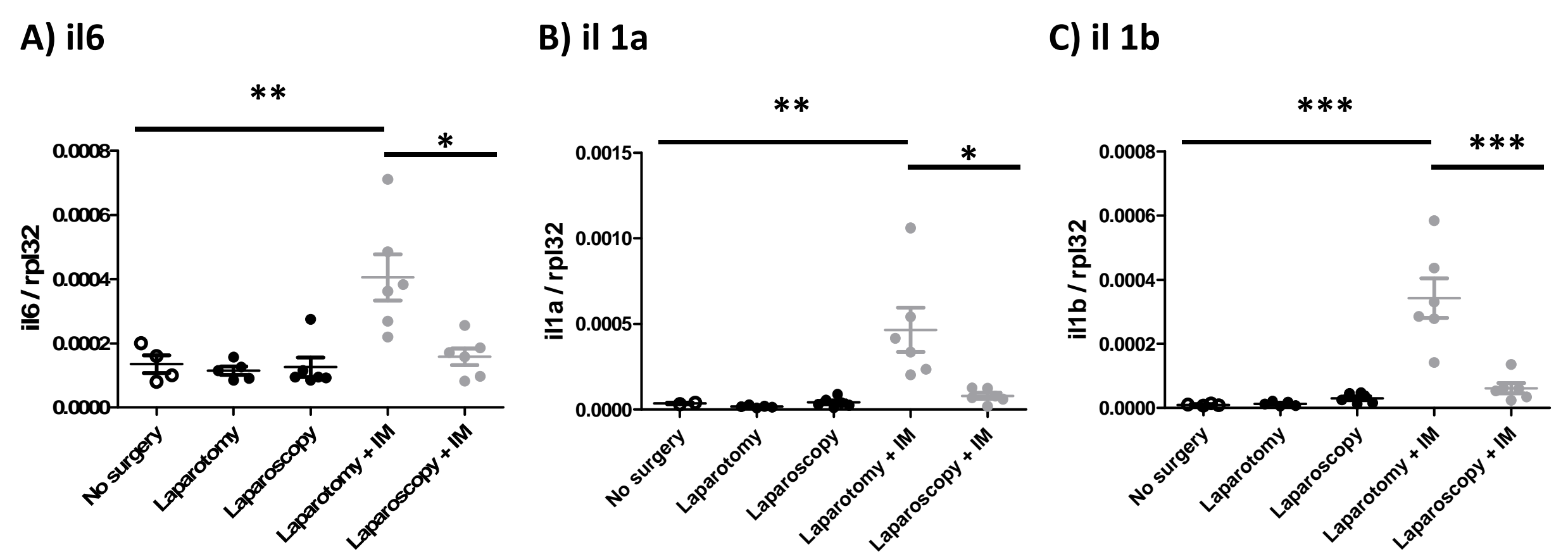




\section{Figure 5}

A) Tolerance to solid food

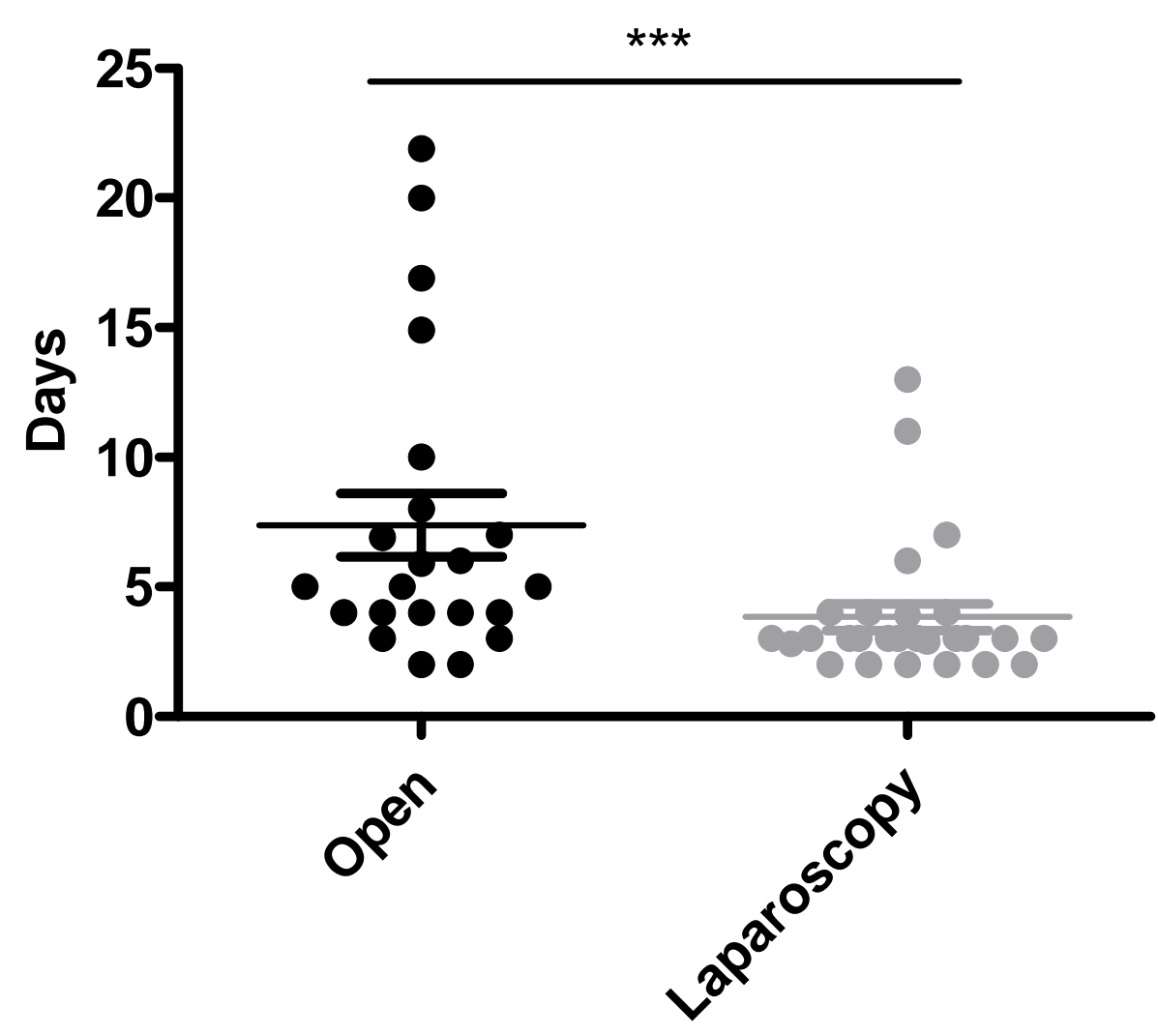

B) Hospital discharge

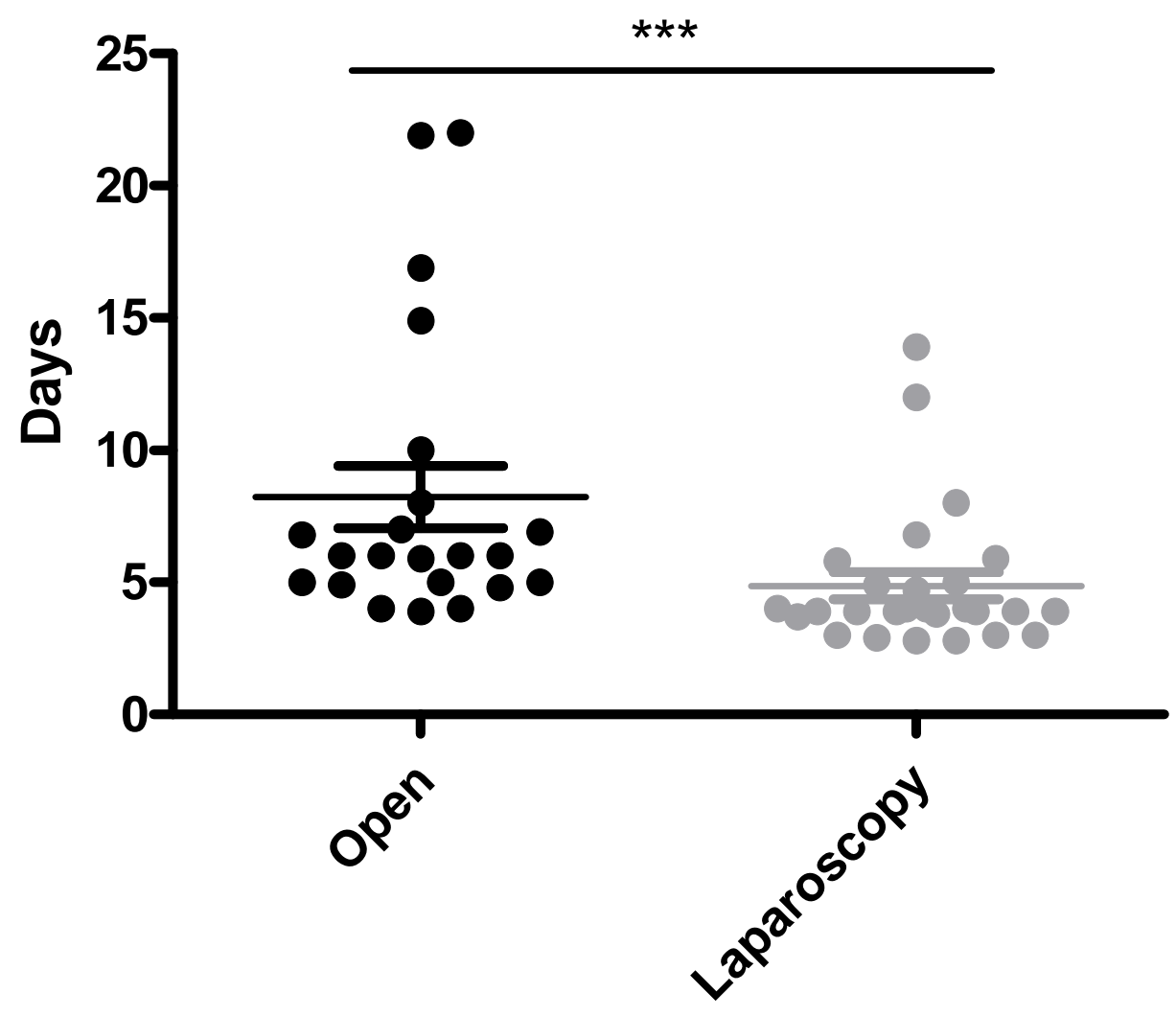



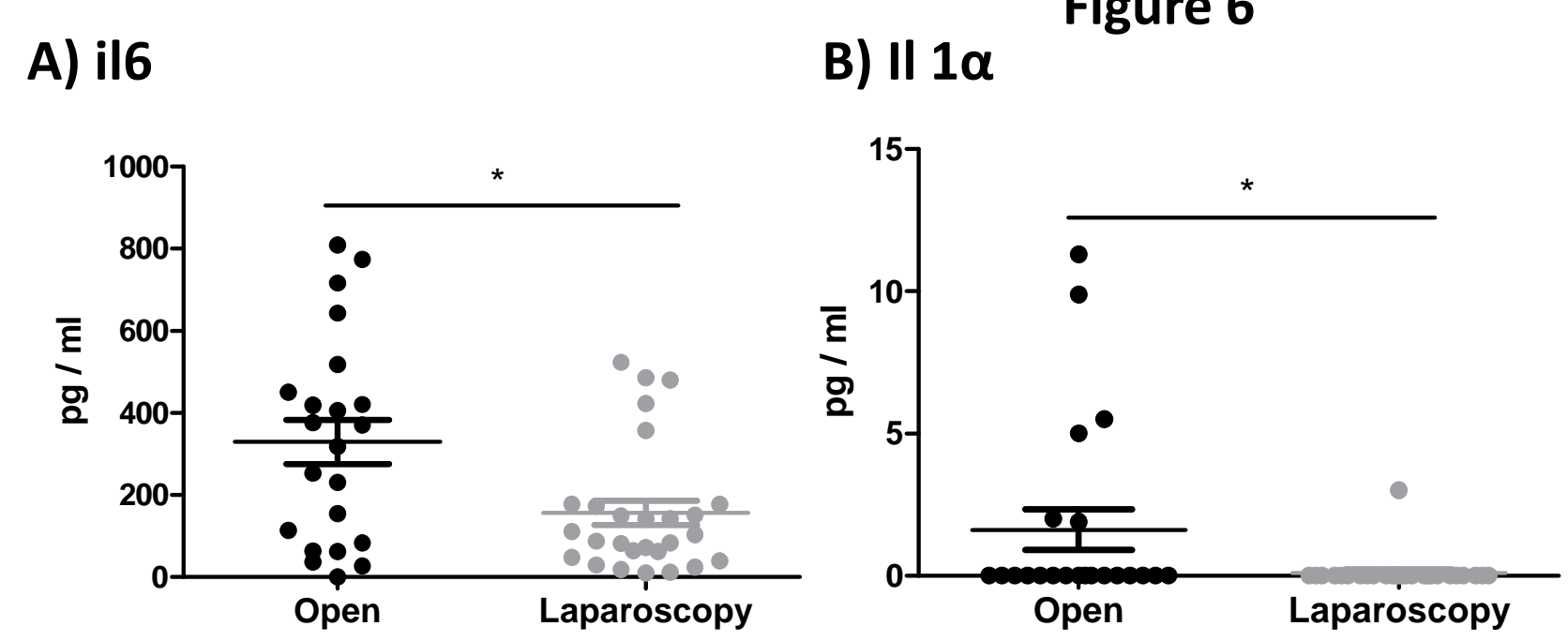

\section{C) II $1 \beta$}

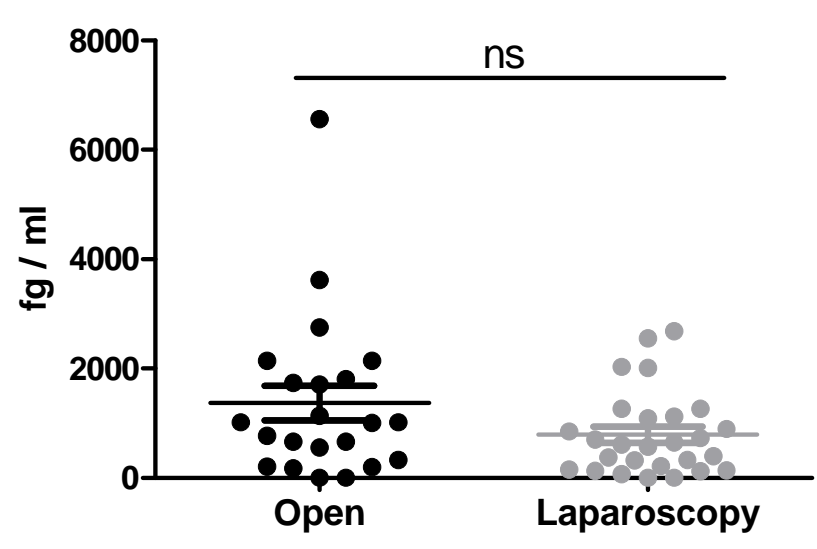

\section{D) il8}

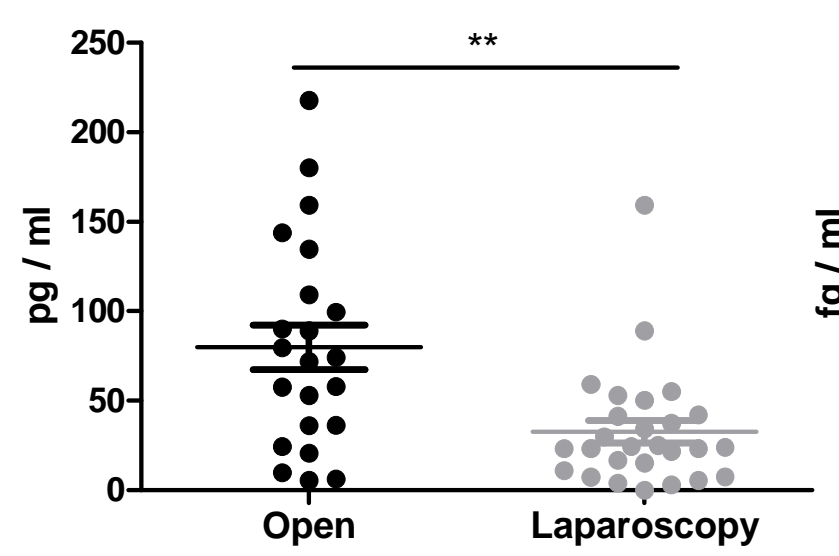

E) II $12 p 70$

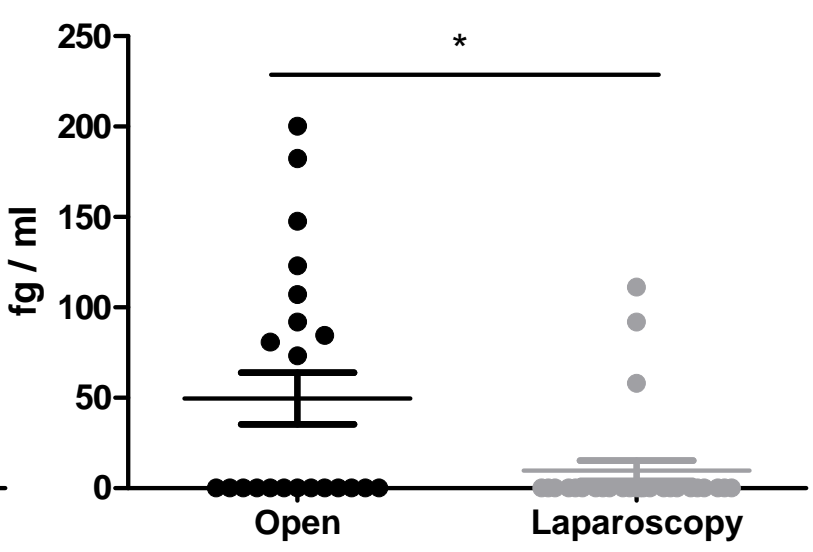

F) TNF $\alpha$

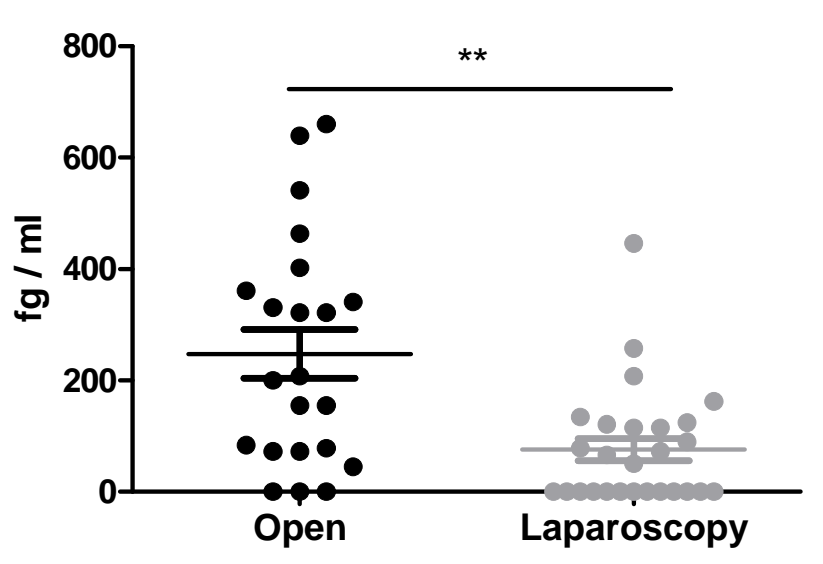

